\title{
FURTHER EXTENSION OF EXTENDED FRACTIONAL DERIVATIVE OPERATOR OF RIEMANN-LIOUVILLE
}

\author{
GAUHAR RAHMAN, SHAHID MUBEEN, KOTTAKKARAN SOOPPY NISAR*
}

\begin{abstract}
The main objective of this present paper is to establish the extension of an extended fractional derivative operator by using an extended beta function recently defined by Parmar et al. by considering the Bessel functions in its kernel. Also, we give some results related to the newly defined fractional operator such as Mellin transform and relations to extended hypergeometric and Appell's function via generating functions.
\end{abstract}

\section{INTRODUCTION}

Recently, the application and importance of fractional calculus have been paid more attention. In the field of mathematical analysis, the fractional calculus is a more helpful tool to find out differentials and integrals with the real numbers or with the complex numbers powers of the fractional calculus. Various extensions and generalization of fractional derivative operators are recently investigated by the researchers (see $[6,8,12,16,17])$.

We begin with the definition of Euler's beta function

$$
\beta\left(\sigma_{1}, \sigma_{2}\right)=\int_{0}^{\infty} t^{\sigma_{1}-1}(1-t)^{\sigma_{2}-1} d t,\left(\Re\left(\sigma_{1}\right)>0, \Re\left(\sigma_{2}\right)>0\right),
$$

and its relation with gamma function is given by

$$
\beta\left(\sigma_{1}, \sigma_{2}\right)=\frac{\Gamma\left(\sigma_{1}\right) \Gamma\left(\sigma_{2}\right)}{\Gamma\left(\sigma_{1}+\sigma_{2}\right)} .
$$

The Gauss hypergeometric and the confluent hypergeometric functions which are respectively defined by(see [15])

and

$$
\begin{array}{r}
{ }_{2} F_{1}\left(\sigma_{1}, \sigma_{2} ; \sigma_{3} ; z\right)=\sum_{n=0}^{\infty} \frac{\left(\sigma_{1}\right)_{n}\left(\sigma_{2}\right)_{n}}{\left(\sigma_{3}\right)_{n}} \frac{z^{n}}{n !},(|z|<1), \\
\left(\sigma_{1}, \sigma_{2}, \sigma_{3} \in \mathbb{C} \text { and } \sigma_{3} \neq 0,-1,-2,-3, \cdots\right),
\end{array}
$$

$$
\begin{aligned}
& { }_{1} \Phi_{1}\left(\sigma_{2} ; \sigma_{3} ; z\right)=\sum_{n=0}^{\infty} \frac{\left(\sigma_{2}\right)_{n}}{\left(\sigma_{3}\right)_{n}} \frac{z^{n}}{n !},(|z|<1) \\
& \left(\sigma_{2}, \sigma_{3} \in \mathbb{C} \text { and } \sigma_{3} \neq 0,-1,-2,-3, \cdots\right)
\end{aligned}
$$

2010 Mathematics Subject Classification. 33C15, 33C05.

Key words and phrases. hypergeometric function; extended hypergeometric function; mellin transform; fractional derivative; appell's function.

*Corresponding author. 
The Appell's series or bivariate hypergeometric series is defined by

$$
F_{1}\left(\sigma_{1}, \sigma_{2}, \sigma_{3} ; \sigma_{4} ; x, y\right)=\sum_{m, n=0}^{\infty} \frac{\left(\sigma_{1}\right)_{m+n}\left(\sigma_{2}\right)_{m}\left(\sigma_{3}\right)_{n} x^{m} y^{n}}{\left(\sigma_{4}\right)_{m+n} m ! n !}
$$

for all $\sigma_{1}, \sigma_{2}, \sigma_{3}, \sigma_{4} \in \mathbb{C}, \sigma_{4} \neq 0,-1,-2,-3, \cdots, \quad|x|<1,|y|<1$.

The integral representation of (1.2), (1.3) and (1.4) are respectively defined by

and

$$
\begin{gathered}
{ }_{2} F_{1}\left(\sigma_{1}, \sigma_{2} ; \sigma_{3} ; z\right)=\frac{\Gamma\left(\sigma_{3}\right)}{\Gamma\left(\sigma_{2}\right) \Gamma\left(\sigma_{3}-\sigma_{2}\right)} \int_{0}^{1} t^{\sigma_{2}-1}(1-t)^{\sigma_{3}-\sigma_{2}-1}(1-z t)^{-\sigma_{1}} d t, \\
\left(\Re\left(\sigma_{3}\right)>\Re\left(\sigma_{2}\right)>0,|\arg (1-z)|<\pi\right),
\end{gathered}
$$

Chaudhry et al. [2] introduced the extended beta function which is defined by

$$
\beta\left(\sigma_{1}, \sigma_{2} ; p\right)=\beta_{p}\left(\sigma_{1}, \sigma_{2}\right)=\int_{0}^{\infty} t^{\sigma_{1}-1}(1-t)^{\sigma_{2}-1} e^{-\frac{p}{t(1-t)}} d t
$$

$$
\begin{aligned}
F_{1}\left(\sigma_{1}, \sigma_{2}, \sigma_{3} ; \sigma_{4} ; x, y\right) & =\frac{\Gamma\left(\sigma_{4}\right)}{\Gamma\left(\sigma_{1}\right) \Gamma\left(\sigma_{4}-\sigma_{1}\right)} \\
& \times \int_{0}^{1} t^{\sigma_{1}-1}(1-t)^{\sigma_{4}-\sigma_{1}-1}(1-x t)^{-\sigma_{2}}(1-y t)^{-\sigma_{3}} d t
\end{aligned}
$$

(where $\Re(p)>0, \Re\left(\sigma_{1}\right)>0, \Re\left(\sigma_{2}\right)>0$ ). When $p=0$, then $\beta\left(\sigma_{1}, \sigma_{2} ; 0\right)=\beta\left(\sigma_{1}, \sigma_{2}\right)$.

The extended hypergeometric and confluent hypergeometric functions is defined in [3] as follows:

$$
F_{p}\left(\sigma_{1}, \sigma_{2} ; \sigma_{3} ; z\right)=\sum_{n=0}^{\infty} \frac{\beta_{p}\left(\sigma_{2}+n, \sigma_{3}-\sigma_{2}\right)}{\beta\left(\sigma_{2}, \sigma_{3}-\sigma_{2}\right)}\left(\sigma_{1}\right)_{n} \frac{z^{n}}{n !}
$$

and

$$
\Phi_{p}\left(\sigma_{2} ; \sigma_{3} ; z\right)=\sum_{n=0}^{\infty} \frac{\beta_{p}\left(\sigma_{2}+n, \sigma_{3}-\sigma_{2}\right)}{\beta\left(\sigma_{2}, \sigma_{3}-\sigma_{2}\right)} \frac{z^{n}}{n !}
$$

where $p \geq 0$.

Also, in [3] authors defined the following integral representations of extended hypergeometric and confluent hypergeometric functions as

$$
\begin{aligned}
F_{p}\left(\sigma_{1}, \sigma_{2} ; \sigma_{3} ; z\right) & =\frac{1}{\beta\left(\sigma_{2}, \sigma_{3}-\sigma_{2}\right)} \\
& \times \int_{0}^{1} t^{\sigma_{2}-1}(1-t)^{\sigma_{3}-\sigma_{2}-1}(1-z t)^{-\sigma_{1}} \exp \left(\frac{-p}{t(1-t)}\right) d t, \\
& \left(p \geq 0, \Re\left(\sigma_{3}\right)>\Re\left(\sigma_{2}\right)>0,|\arg (1-z)|<\pi\right),
\end{aligned}
$$


and

$$
\begin{gathered}
\Phi_{p}\left(\sigma_{2}, \sigma_{3} ; \gamma ; z\right)=\frac{1}{\beta\left(\sigma_{2}, \sigma_{3}-\sigma_{2}\right)} \int_{0}^{1} t^{\sigma_{2}-1}(1-t)^{\sigma_{3}-\sigma_{2}-1} \exp \left(z t-\frac{p}{t(1-t)}\right) d t, \\
\left(p \geq 0, \Re\left(\sigma_{3}\right)>\Re\left(\sigma_{2}\right)>0\right) .
\end{gathered}
$$

The extended Appell's function is defined by (see [10])

$$
F_{1}\left(\sigma_{1}, \sigma_{2}, \sigma_{3} ; \sigma_{4} ; x, y ; p\right)=\sum_{n=0}^{\infty} \frac{\beta_{p}\left(\sigma_{1}+m+n, \sigma_{4}-\sigma_{1}\right)}{\beta\left(\sigma_{1}, \sigma_{4}-\sigma_{1}\right)}\left(\sigma_{2}\right)_{m}\left(\sigma_{3}\right)_{n} \frac{x^{m} y^{n}}{m ! n !}
$$

where $p \geq 0$ and its integral representation by

$$
\begin{aligned}
F_{1}\left(\sigma_{1}, \sigma_{2}, \sigma_{3} ; \sigma_{4} ; x, y ; p\right) & =\frac{1}{\beta\left(\sigma_{1}, \sigma_{4}-\sigma_{1}\right)} \int_{0}^{1} t^{\sigma_{1}-1}(1-t)^{\sigma_{4}-\sigma_{1}-1}(1-x t)^{-\sigma_{2}}(1-y t)^{-\sigma_{3}} \\
& \times \exp \left(\frac{-p}{t(1-t)}\right) d t \\
& \left(p \geq 0, \Re\left(\sigma_{4}\right)>\Re\left(\sigma_{1}\right)>0,|\arg (1-x)|<\pi,|\arg (1-y)|<\pi\right) .
\end{aligned}
$$

It is clear that when $p=0$, then the equations (1.9)-(1.14) reduce to the well known hypergeometric, confluent hypergeometric and Appell's series and their integral representation respectively.

For various extensions and generalization the readers may follow the recent work of researchers (see e.g., [1, 4, 9]). Parmar et al. [14] introduced the following extended beta function as

$$
\beta_{v}\left(\sigma_{1}, \sigma_{2} ; p\right)=\sqrt{\frac{2 p}{\pi}} \int_{0}^{1} t^{\sigma_{1}-\frac{3}{2}}(1-t)^{y-\frac{3}{2}} K_{v+\frac{1}{2}}\left(\frac{p}{t(1-t)}\right) d t
$$

where $K_{v+\frac{1}{2}}($.$) is the modified Bessel function of order v+\frac{1}{2}$. Clearly, when $v=0$ then (1.15) reduces to (1.8) by using the fact that $K_{\frac{1}{2}}(z)=\sqrt{\frac{\pi}{2 z}} e^{-z}$. Also, the following extended hypergeometric and confluent hypergeometric functions and their integral representation respectively as (see, [14]):

$$
\begin{gathered}
F_{p, v}\left(\sigma_{1}, \sigma_{2} ; \sigma_{3} ; z\right)=\sum_{n=0}^{\infty}\left(\sigma_{1}\right)_{n} \frac{\beta_{v}\left(\sigma_{2}+n, \sigma_{3}-\sigma_{2} ; p\right)}{\beta\left(\sigma_{2}, \sigma_{3}-\sigma_{2}\right)} \frac{z^{n}}{n !} \\
\left(p, v \geq 0, \Re\left(\sigma_{3}>\sigma_{2}>0,|z|<1\right) .\right. \\
\Phi_{p, v}\left(\sigma_{2} ; \sigma_{3} ; z\right)=\sum_{n=0}^{\infty} \frac{\beta_{v}\left(\sigma_{2}+n, \sigma_{3}-\sigma_{2} ; p\right)}{\beta\left(\sigma_{2}, \sigma_{3}-\sigma_{2}\right)} \frac{z^{n}}{n !} \\
F_{p, v}\left(\sigma_{1}, \sigma_{2} ; \sigma_{3} ; z\right)=\sqrt{\frac{2 p}{\pi}} \frac{1}{\beta\left(\sigma_{2}, \sigma_{3}-\sigma_{2}\right)} \\
\times \int_{0}^{1} t^{\sigma_{2}-\frac{3}{2}}(1-t)^{\sigma_{3}-\sigma_{2}-\frac{3}{2}}(1-z t)^{-\sigma_{1}} K_{v+\frac{1}{2}}\left(\frac{p}{t(1-t)}\right) d t
\end{gathered}
$$


and

$$
\left(p, v \geq 0, \Re\left(\sigma_{3}>\sigma_{2}>0,|\arg (1-z)|<\pi\right)\right. \text {. }
$$

$$
\begin{aligned}
& \Phi_{p, v}\left(\sigma_{2} ; \sigma_{3} ; z\right)= \sqrt{\frac{2 p}{\pi}} \frac{1}{\beta\left(\sigma_{2}, \sigma_{3}-\sigma_{2}\right)} \\
& \times \int_{0}^{1} t^{\sigma_{2}-\frac{3}{2}}(1-t)^{\sigma_{3}-\sigma_{2}-\frac{3}{2}} \exp (z t) K_{v+\frac{1}{2}}\left(\frac{p}{t(1-t)}\right) d t, \\
& \quad\left(p, v \geq 0, \Re\left(\sigma_{3}>\sigma_{2}>0\right) .\right.
\end{aligned}
$$

They also obtained the following transformation formula for extended confluent hypergeometric function

$$
\Phi_{p, v}\left(\sigma_{2}, \sigma_{3} ; \gamma ; z\right)=e^{z} \Phi_{p, v}\left(\sigma_{3}-\sigma_{2} ; \sigma_{3} ;-z\right) .
$$

It is clear that, when $v=0$ then the equations (1.16)-(1.19) reduce to the extended hypergeometric, confluent hypergeometric functions and their integral representations defined in (1.9)-(1.12) respectively by using the fact that $K_{\frac{1}{2}}(z)=\sqrt{\frac{\pi}{2 z}} e^{-z}$.

Recently Dar and Paris [5] have introduced the following Appell's hypergeometric function by

$$
\begin{aligned}
F_{1, p, v}\left(\sigma_{1}, \sigma_{2}, \sigma_{3} ; \sigma_{4} ; x, y\right) & =F_{1, v}\left(\sigma_{1}, \sigma_{2}, \sigma_{3} ; \sigma_{4} ; x, y ; p\right) \\
= & \sum_{m, n=0}\left(\sigma_{2}\right)_{m}\left(\sigma_{3}\right)_{n} \frac{\beta\left(\sigma_{1}+m+n, \sigma_{4}-\sigma_{1}\right)}{\beta\left(\sigma_{1}, \sigma_{4}-\sigma_{1}\right)} \frac{x^{m} y^{n}}{n ! m !}
\end{aligned}
$$

where $|x|<1,|y|<1, \sigma_{1}, \sigma_{2}, \sigma_{3}, \sigma_{4} \in \mathbb{C}, \sigma_{4} \neq=0,-1,-2,-3, \cdots$.

In the same paper, they [5] defined its integral representation as:

$$
\begin{aligned}
F_{1, p, v}\left(\sigma_{1}, \sigma_{2}, \sigma_{3} ; \sigma_{4} ; x, y\right) & =\sqrt{\frac{2 p}{\pi}} \frac{1}{\beta\left(\sigma_{1}, \sigma_{4}-\sigma_{1}\right)} \int_{0}^{1} t^{\sigma_{1}-\frac{3}{2}}(1-t)^{\sigma_{4}-\sigma_{1}-\frac{3}{2}}(1-t x)^{-\sigma_{2}}(1-t y)^{-\sigma_{3}} \\
\times & K_{v+\frac{1}{2}}\left(\frac{p}{t(1-t)}\right) d t
\end{aligned}
$$

where $\Re(p) \geq 0, v \geq 0, \Re\left(\sigma_{4}\right)>\Re\left(\sigma_{1}\right)>0,|\arg (1-x)|<\pi$ and $|\arg (1-y)|<\pi$. Obviously, when $v=0$ in (1.21) and (1.22) then we get the extended Appell function and its integral representation (see , (1.13) and (1.14)) by using the fact that $K_{\frac{1}{2}}(z)=$ $\sqrt{\frac{\pi}{2 z}} e^{-z}$. Similarly, when $v=p=0$ then (1.21) and (1.22) reduce to the well-known classical Appell's function and its integral representation.

\section{Extension of FRACTiOnal DERIVATIVE operator}

In this section, we define further extension of extended Riemann-Liouville fractional derivative.

Definition 2.1. The well-known Riemann-Liouville fractional derivative of order $\mu$ is defined by

$$
\mathfrak{D}_{x}^{\mu}\{f(x)\}=\frac{1}{\Gamma(-\mu)} \int_{0}^{x} f(t)(x-t)^{-\mu-1} d t, \Re(\mu)>0
$$


FURTHER EXTENSION OF EXTENDED FRACTIONAL DERIVATIVE OPERATOR...

For the case $m-1<\Re(\mu)<m$ where $m=1,2, \cdots$, it follows

$$
\begin{aligned}
\mathfrak{D}_{x}^{\mu}\{f(z)\} & =\frac{d^{m}}{d x^{m}} \mathfrak{D}_{x}^{\mu-m}\{f(x)\} \\
& =\frac{d^{m}}{d x^{m}}\left\{\frac{1}{\Gamma(-\mu+m)} \int_{0}^{x} f(t)(x-t)^{-\mu+m-1} d t\right\}, \Re(\mu)>0 .
\end{aligned}
$$

Definition 2.2. (see [10]) The extended Riemann-Liouville fractional derivative of order $\mu$ is defined by

$$
\mathfrak{D}_{x}^{\mu}\{f(x) ; p\}=\frac{1}{\Gamma(-\mu)} \int_{0}^{x} f(t)(x-t)^{-\mu-1} \exp \left(-\frac{p x^{2}}{t(x-t)}\right) d t, \Re(\mu)>0 .
$$

For the case $m-1<\Re(\mu)<m$ where $m=1,2, \cdots$, it follows

$$
\begin{aligned}
\mathfrak{D}_{x}^{\mu}\{f(z) ; p\} & =\frac{d^{m}}{d x^{m}} \mathfrak{D}_{x}^{\mu-m}\{f(x) ; p\} \\
& =\frac{d^{m}}{d x^{m}}\left\{\frac{1}{\Gamma(-\mu+m)} \int_{0}^{x} f(t)(x-t)^{-\mu+m-1} \exp \left(-\frac{p x^{2}}{t(x-t)}\right) d t\right\}, \Re(\mu)>0 .
\end{aligned}
$$

Definition 2.3. (see [1])

$$
\mathfrak{D}_{x}^{\mu}\{f(x) ; p, q\}=\frac{1}{\Gamma(-\mu)} \int_{0}^{x} f(t)(x-t)^{-\mu-1} \cdot \exp \left(-\frac{p x}{t}-\frac{q x}{(x-t)}\right) d t, \Re(\mu)>0 .
$$

For the case $m-1<\Re(\mu)<m$ where $m=1,2, \cdots$, it follows

$$
\begin{aligned}
\mathfrak{D}_{x}^{\mu}\{f(z) ; p, q\} & =\frac{d^{m}}{d x^{m}} \mathfrak{D}_{x}^{\mu-m}\{f(x) ; p, q\} \\
& =\frac{d^{m}}{d x^{m}}\left\{\frac{1}{\Gamma(-\mu+m)} \int_{0}^{x} f(t)(x-t)^{-\mu+m-1}\right. \\
& \left.\times \exp \left(-\frac{p x}{t}-\frac{q x}{(x-t)}\right) d t\right\}, \Re(\mu)>0 .
\end{aligned}
$$

Recently Rahman et al. [16] defined a new extension Riemann-Liouville fractional derivative of order $\mu$ as:

Definition 2.4. The extension of extended Riemann-Liouville fractional derivative of order $\mu$ as

$$
\begin{aligned}
\mathfrak{D}_{x}^{\mu}\{f(x) ; p, q, \lambda, \rho\} & =\frac{1}{\Gamma(-\mu)} \int_{0}^{x} f(t)(x-t)^{-\mu-1} \\
& \times{ }_{1} F_{1}\left[\lambda ; \rho ;-\frac{p x}{t}\right]{ }_{1} F_{1}\left[\lambda ; \rho ;-\frac{q x}{(x-t)}\right] d t, \Re(\mu)>0 .
\end{aligned}
$$

For the case $m-1<\Re(\mu)<m$ where $m=1,2, \cdots$, it follows

$$
\begin{aligned}
& \mathfrak{D}_{x}^{\mu}\{f(z) ; p, q, \lambda, \rho\}=\frac{d^{m}}{d x^{m}} \mathfrak{D}_{x}^{\mu-m}\{f(x) ; p, q, \lambda, \rho\} \\
& =\frac{d^{m}}{d x^{m}}\left\{\frac{1}{\Gamma(-\mu+m)} \int_{0}^{x} f(t)(x-t)_{1}^{-\mu+m-1} F_{1}\left[\lambda ; \rho ;-\frac{p x}{t}\right]{ }_{1} F_{1}\left[\lambda ; \rho ;-\frac{q x}{(x-t)}\right] d t\right\},
\end{aligned}
$$

where $\Re(\mu)>0, \Re(p)>0$ and $\Re(q)>0$. 
Next, we give an extension of extended Riemann-Liouville fractional derivative operator (2.2) of order $\mu$ as:

Definition 2.5. The extension of extended Riemann-Liouville fractional derivative of order $\mu$ as

$$
\mathfrak{D}_{x}^{\mu}\{f(x) ; p, v\}=\sqrt{\frac{2 p}{\pi}} \frac{1}{\Gamma(-\mu)} \int_{0}^{x} f(t)(x-t)^{-\mu-\frac{3}{2}} K_{v+\frac{1}{2}}\left(\frac{p x^{2}}{t(x-t)}\right) d t, \Re(\mu)>0 .
$$

For the case $m-1<\Re(\mu)<m$ where $m=1,2, \cdots$, it follows

$$
\begin{aligned}
& \mathfrak{D}_{x}^{\mu}\{f(x) ; p, v\}=\frac{d^{m}}{d x^{m}} \mathfrak{D}_{x}^{\mu-m}\{f(x) ; p, v\} \\
& =\frac{d^{m}}{d x^{m}}\left\{\sqrt{\frac{2 p}{\pi}} \frac{1}{\Gamma(-\mu+m)} \int_{0}^{x} f(t)(x-t)^{-\mu+m-\frac{3}{2}} K_{v+\frac{1}{2}}\left(\frac{p x^{2}}{t(x-t)}\right) d t\right\},
\end{aligned}
$$

where $\Re(\mu)>0, \Re(p)>0$ and $v \geq 0$.

\section{Remark 2.1. Clearly}

(i) If $v=0$, then definition 2.5 reduces to extended fractional derivative defined in definition 2.2 by using the fact that $K_{\frac{1}{2}}(z)=\sqrt{\frac{\pi}{2 z}} e^{-z}$.

(ii) If $v=p=0$, then definition 2.5 reduces to the Riemann-Liouville fractional derivative defined in definition 2.1.

Now, we prove some theorem involving the extension of fractional derivative.

Theorem 2.1. The following formula hold true,

$$
\mathfrak{D}_{z}^{\mu}\left\{z^{\eta} ; p, v\right\}=\frac{\beta_{p, v}\left(\eta+\frac{3}{2},-\mu\right)}{\Gamma(-\mu)} z^{\eta-\mu-\frac{1}{2}}, \Re(\mu)>0 .
$$

Proof. From (2.9), we have

$$
\mathfrak{D}_{z}^{\mu}\left\{z^{\eta} ; p, v\right\}=\sqrt{\frac{2 p}{\pi}} \frac{1}{\Gamma(-\mu)} \int_{0}^{z} t^{\eta}(z-t)^{-\mu-\frac{3}{2}} K_{v+\frac{1}{2}}\left(\frac{p z^{2}}{t(z-t)}\right) d t .
$$

Substituting $t=u z$ in (2.12), we get

$$
\begin{aligned}
\mathfrak{D}_{z}^{\mu}\left\{z^{\eta} ; p, v\right\} & =\sqrt{\frac{2 p}{\pi}} \frac{1}{\Gamma(-\mu)} \int_{0}^{1}(u z)^{\eta}(z-u z)^{-\mu-\frac{3}{2}} K_{v+\frac{1}{2}}\left(\frac{p z^{2}}{u z(z-u z)}\right) d u \\
& =\frac{z^{\eta-\mu-\frac{1}{2}} \sqrt{\frac{2 p}{\pi}}}{\Gamma(-\mu)} \int_{0}^{1} u^{\eta}(1-u)^{-\mu-\frac{3}{2}} K_{v+\frac{1}{2}}\left(\frac{p}{u(1-u)}\right) d u,
\end{aligned}
$$

by applying definition (1.15) to the above equation, we get the desired result.

Theorem 2.2. Let $\Re(\mu)>0$ and suppose that the function $f(z)$ is analytic at the origin with its Maclaurin expansion given by $f(z)=\sum_{n=0}^{\infty} a_{n} z^{n}$ where $|z|<\delta$ for some $\delta \in \mathbb{R}^{+}$. Then

$$
\mathfrak{D}_{z}^{\mu}\{f(z) ; p, v\}=\sum_{n=0}^{\infty} a_{n} \mathfrak{D}_{z}^{\mu}\left\{z^{n} ; p, v\right\}
$$


Proof. Using the series expansion of the function $f(z)$ in $(2.7)$ gives

$$
\mathfrak{D}_{z}^{\mu}\{f(z) ; p, v\}=\sqrt{\frac{2 p}{\pi}} \frac{1}{\Gamma(-\mu)} \int_{0}^{z} \sum_{n=0}^{\infty} a_{n} t^{n}(z-t)^{-\mu-\frac{3}{2}} K_{v+\frac{1}{2}}\left(\frac{p z^{2}}{t(z-t)}\right) d t .
$$

As the series is uniformly convergent on any closed disk centered at the origin with its radius smaller then $\delta$, therefore the series so does on the line segment from 0 to a fixed $z$ for $|z|<\delta$. Thus it guarantee terms by terms integration as follows

$$
\begin{aligned}
\mathfrak{D}_{z}^{\mu}\{f(z) ; p, v\} & =\sum_{n=0}^{\infty} a_{n}\left\{\sqrt{\frac{2 p}{\pi}} \frac{1}{\Gamma(-\mu)} \int_{0}^{z} t^{n}(z-t)^{-\mu-\frac{3}{2}} K_{v+\frac{1}{2}}\left(\frac{p z^{2}}{t(z-t)}\right) d t\right\} \\
& =\sum_{n=0}^{\infty} a_{n} \mathfrak{D}_{z}^{\mu}\left\{z^{n} ; p, v\right\},
\end{aligned}
$$

which is the required proof.

Theorem 2.3. The following result holds true:

$$
\mathfrak{D}_{z}^{\eta-\mu}\left\{z^{\eta-\frac{3}{2}}(1-z)^{-\beta} ; p, v\right\}=\frac{\Gamma(\eta)}{\Gamma(\mu)} z_{2}^{\mu-2}{ }_{2} F_{1 ; p, v}^{\lambda, \rho}(\beta, \eta ; \mu ; z),
$$

where $\Re(\mu)>\Re(\eta)>0$ and $|z|<1$.

Proof. By direct calculation, we have

$$
\begin{aligned}
\mathfrak{D}_{z}^{\eta-\mu}\left\{z^{\eta-\frac{3}{2}}(1-z)^{-\beta} ; p, v\right\} & =\sqrt{\frac{2 p}{\pi}} \frac{1}{\Gamma(\mu-\eta)} \int_{0}^{z} t^{\eta-\frac{3}{2}}(1-t)^{-\beta}(z-t)^{\mu-\eta-\frac{3}{2}} \\
& \times K_{v+\frac{1}{2}}\left(\frac{p z^{2}}{t(z-t)}\right) d t \\
& =\sqrt{\frac{2 p}{\pi}} \frac{z^{\mu-\eta-\frac{3}{2}}}{\Gamma(\mu-\eta)} \int_{0}^{z} t^{\eta-\frac{3}{2}}(1-t)^{-\beta}\left(1-\frac{t}{z}\right)^{\mu-\eta-\frac{3}{2}} \\
& \times K_{v+\frac{1}{2}}\left(\frac{p z^{2}}{t(z-t)}\right) d t .
\end{aligned}
$$

Substituting $t=z u$ in the above equation, we get

$$
\begin{aligned}
\mathfrak{D}_{z}^{\eta-\mu}\left\{z^{\eta-\frac{3}{2}}(1-z)^{-\beta} ; p, v\right\} & =\frac{z^{\mu-2}}{\Gamma(\mu-\eta)} \sqrt{\frac{2 p}{\pi}} \int_{0}^{1} u^{\eta-\frac{3}{2}}(1-u z)^{-\beta}(1-u)^{\mu-\eta-\frac{3}{2}} \\
& \times K_{v+\frac{1}{2}}\left(\frac{p}{u(1-u)}\right) d u .
\end{aligned}
$$

Using (1.16) and after simplification we get the required proof.

Theorem 2.4. The following result holds true:

$$
\mathfrak{D}_{z}^{\eta-\mu}\left\{z^{\eta-\frac{3}{2}}(1-a z)^{-\alpha}(1-b z)^{-\beta} ; p, v\right\}=\frac{\Gamma(\eta)}{\Gamma(\mu)} z^{\mu-2} F_{1}(\eta, \alpha, \beta ; \mu ; a z, b z ; p, v),
$$

where $\Re(\mu)>\Re(\eta)>0, \Re(\alpha)>0, \Re(\beta)>0,|a z|<1$ and $|b z|<1$. 
Proof. To prove(2.15), we use the following power series expansion

$$
(1-a z)^{-\alpha}(1-b z)^{-\beta}=\sum_{m=0}^{\infty} \sum_{n=0}^{\infty}(\alpha)_{m}(\beta)_{n} \frac{(a z)^{m}}{m !} \frac{(b z)^{n}}{n !} .
$$

Now, applying Theorem 2.3, we obtain

$$
\begin{aligned}
& \mathfrak{D}_{z}^{\eta-\mu}\left\{z^{\eta-\frac{3}{2}}(1-a z)^{-\alpha}(1-b z)^{-\beta} ; p, v\right\} \\
& =\sum_{m=0}^{\infty} \sum_{n=0}^{\infty}(\alpha)_{m}(\beta)_{n} \frac{(a)^{m}}{m !} \frac{(b)^{n}}{n !} \mathfrak{D}_{z}^{\eta-\mu}\left\{z^{\eta+m+n-\frac{3}{2}} ; p, v\right\} \\
& =\sum_{m=0}^{\infty} \sum_{n=0}^{\infty}(\alpha)_{m}(\beta)_{n} \frac{(a)^{m}}{m !} \frac{(b)^{n}}{n !} \frac{\beta_{p, v}(\eta+m+n, \mu-\eta)}{\Gamma(\mu-\eta)} z^{\mu+m+n-2}
\end{aligned}
$$

Now, applying (1.21), we get

$$
\mathfrak{D}_{z}^{\eta-\mu}\left\{z^{\eta-\frac{3}{2}}(1-a z)^{-\alpha}(1-b z)^{-\beta} ; p, v\right\}=\frac{\Gamma(\eta)}{\Gamma(\mu)} z^{\mu-2} F_{1}(\eta, \alpha, \beta ; \mu ; a z, b z ; p, v) .
$$

Theorem 2.5. The following Mellin transform formula holds true:

$$
M\left\{\mathfrak{D}_{z ; p, v}^{\mu}\left(z^{\eta}\right) ; p \rightarrow r\right\}=\frac{z^{\eta-\mu-\frac{1}{2}} 2^{r-1} \Gamma\left(\frac{r-v}{2}\right) \Gamma\left(\frac{r+v+1}{2}\right)}{\sqrt{\pi} \Gamma(-\mu)} \beta(\eta+r, r-\mu),
$$

where $\Re(\eta)>-1, \Re(\mu)>0, \Re(r)>0$.

Proof. Applying the Mellin transform on definition (2.9), we have

$$
\begin{aligned}
& M\left\{\mathfrak{D}_{z ; p, v}^{\mu}\left(z^{\eta}\right) ; p \rightarrow r\right\}=\int_{0}^{\infty} p^{r-1} \mathfrak{D}_{z ; p, v}^{\mu}\left(z^{\eta}\right) d p \\
& =\sqrt{\frac{2 p}{\pi}} \frac{1}{\Gamma(-\mu)} \int_{0}^{\infty} p^{r-1}\left\{\int_{0}^{z} t^{\eta}(z-t)^{-\mu-\frac{3}{2}} K_{v+\frac{1}{2}}\left(\frac{p z^{2}}{t(z-t)}\right) d t\right\} d p \\
& =\frac{z^{-\mu-\frac{3}{2}} \sqrt{\frac{2 p}{\pi}}}{\Gamma(-\mu)} \int_{0}^{\infty} \int_{0}^{\infty} p^{r-1}\left\{\int_{0}^{z} t^{\eta}\left(1-\frac{t}{z}\right)^{-\mu-\frac{3}{2}} \times K_{v+\frac{1}{2}}\left(\frac{p z^{2}}{t(z-t)}\right) d t\right\} d p \\
& =\frac{z^{\eta-\mu-\frac{1}{2}} \sqrt{\frac{2 p}{\pi}}}{\Gamma(-\mu)} \int_{0}^{\infty} p^{r-1}\left\{\int_{0}^{1} u^{\eta}(1-u)^{-\mu-\frac{3}{2}} K_{v+\frac{1}{2}}\left(\frac{p}{u(1-u)}\right) d u\right\} d p \\
& =\frac{z^{\eta-\mu-\frac{1}{2}} \sqrt{\frac{2}{\pi}}}{\Gamma(-\mu)} \int_{0}^{1} u^{\eta}(1-u)^{-\mu-\frac{3}{2}}\left(\int_{0}^{\infty} p^{r-\frac{1}{2}} K_{v+\frac{1}{2}}\left(\frac{p}{u(1-u)}\right) d p\right) d u .
\end{aligned}
$$

Since

$$
\begin{aligned}
\int_{0}^{\infty} p^{r-\frac{1}{2}} K_{v+\frac{1}{2}}\left(\frac{p}{t(1-t)}\right) d p & =u^{r+\frac{1}{2}}(1-u)^{r+\frac{1}{2}} \int_{0}^{\infty} w^{r-\frac{1}{2}} K_{v+\frac{1}{2}}(w) d w \\
& =u^{r+\frac{1}{2}}(1-u)^{r+\frac{1}{2}} 2^{r-\frac{3}{2}} \Gamma\left(\frac{r-v}{2}\right) \Gamma\left(\frac{r+v+1}{2}\right) .
\end{aligned}
$$


FURTHER EXTENSION OF EXTENDED FRACTIONAL DERIVATIVE OPERATOR...

where $w=\frac{p}{u(1-u)}, \Re(r-v)>0, \Re(r+v)>-1$ (see [14] and [12]). Using (2.18) in (2.17), we obtain

$$
\begin{gathered}
M\left\{\mathfrak{D}_{z ; p, v}^{\mu}\left(z^{\eta}\right) ; p \rightarrow r\right\}=\frac{z^{\eta-\mu-\frac{1}{2}} 2^{r-1} \Gamma\left(\frac{r-v}{2}\right) \Gamma\left(\frac{r+v+1}{2}\right)}{\sqrt{\pi} \Gamma(-\mu)} \int_{0}^{1} u^{\eta+r-1}(1-u)^{r-\mu-1} d u \\
=\frac{z^{\eta-\mu-\frac{1}{2}} 2^{r-1} \Gamma\left(\frac{r-v}{2}\right) \Gamma\left(\frac{r+v+1}{2}\right)}{\sqrt{\pi} \Gamma(-\mu)} \beta(\eta+r, r-\mu),
\end{gathered}
$$

which completes the proof.

Theorem 2.6. The following Mellin transform formula holds true:

$$
\begin{aligned}
M\left\{\mathfrak{D}_{z ; p, v}^{\mu}\left((1-z)^{-\alpha}\right) ; p \rightarrow r\right\} & =\frac{2^{r-1} \Gamma\left(\frac{r-v}{2}\right) \Gamma\left(\frac{r+v+1}{2}\right) z^{-\mu-\frac{1}{2}}}{\sqrt{\pi} \Gamma(-\mu)} \\
& \times \beta(r, r-\mu){ }_{2} F_{1}(\alpha, r ; r-\mu ; z),
\end{aligned}
$$

where $\Re(\alpha)>0, \Re(\mu)<0, \Re(r)>0, \Re(r)>0$ and $|z|<1$.

Proof. Using the power series for $(1-z)^{-\alpha}$ and applying Theorem 2.5 with $\eta=n$, we can write

$$
\begin{aligned}
M\left\{\mathfrak{D}_{z ; p, v}^{\mu}\left((1-z)^{-\alpha}\right) ; p \rightarrow r\right\} & =\sum_{n=0}^{\infty} \frac{(\alpha)_{n}}{n !} M\left\{\mathfrak{D}_{z ; p, v}^{\mu}\left(z^{n}\right) ; p \rightarrow r\right\} \\
& =\frac{2^{r-1} \Gamma\left(\frac{r-v}{2}\right) \Gamma\left(\frac{r+v+1}{2}\right)}{\sqrt{\pi} \Gamma(-\mu)} \sum_{n=0}^{\infty} \frac{(\alpha)_{n}}{n !} \beta(n+r, r-\mu) z^{n-\mu-\frac{1}{2}} \\
& =\frac{2^{r-1} \Gamma\left(\frac{r-v}{2}\right) \Gamma\left(\frac{r+v+1}{2}\right) z^{-\mu-\frac{1}{2}}}{\sqrt{\pi} \Gamma(-\mu)} \sum_{n=0}^{\infty} \beta(n+r, r-\mu) \frac{(\alpha)_{n} z^{n}}{n !} \\
& =\frac{2^{r-1} \Gamma\left(\frac{r-v}{2}\right) \Gamma\left(\frac{r+v+1}{2}\right) z^{-\mu-\frac{1}{2}}}{\sqrt{\pi} \Gamma(-\mu)} \beta(r, r-\mu)_{2} F_{1}(\alpha, r ; r-\mu ; z),
\end{aligned}
$$

which is the required proof.

\section{Generating Relations And some Further Results}

In this section, we derive some generating relations of linear and bilinear type for the extended $(p, v)$-hypergeometric functions.

Theorem 3.1. The following generating relation holds true:

$$
\sum_{n=0}^{\infty} \frac{(\alpha)_{n}}{n !}{ }_{2} F_{1 ; p, v}(\alpha+n, \beta ; \gamma ; z) t^{n}=(1-t)_{2}^{-\alpha} F_{1 ; p, v}\left(\alpha, \beta ; \gamma ; \frac{z}{1-t}\right)
$$

where $|z|<\min (|1,1-t|), \Re(\alpha)>0, \Re(\gamma)>\Re(\beta)>0$.

Proof. Consider the following series identity

$$
[(1-z)-t]^{-\alpha}=(1-t)^{-\alpha}\left[1-\frac{x}{1-t}\right]^{-\alpha} .
$$


Thus, the power series expansion yields

$$
\sum_{n=0}^{\infty} \frac{(\alpha)_{n}}{n !}(1-z)^{-\alpha}\left(\frac{t}{1-z}\right)^{n}=(1-t)^{-\alpha}\left[1-\frac{z}{1-t}\right]^{-\alpha}
$$

Multiplying both sides of (3.2) by $z^{\beta-\frac{3}{2}}$ and then applying the operator $\mathfrak{D}_{z ; p, q}^{\beta-\gamma ; \lambda, \rho}$ on both sides, we have

$$
\mathfrak{D}_{z ; p, v}^{\beta-\gamma}\left[\sum_{n=0}^{\infty} \frac{(\alpha)_{n}}{n !}(1-z)^{-\alpha}\left(\frac{t}{1-z}\right)^{n} z^{\beta-\frac{3}{2}}\right]=(1-t)^{-\alpha} \mathfrak{D}_{z ; p, v}^{\beta-\gamma}\left[z^{\beta-\frac{3}{2}}\left(1-\frac{z}{1-t}\right)^{-\alpha}\right] .
$$

Interchanging the order of summation and the operator $\mathfrak{D}_{z ; p, v}^{\beta-\gamma}$, we have

$$
\sum_{n=0}^{\infty} \frac{(\alpha)_{n}}{n !} \mathfrak{D}_{z ; p, v}^{\beta-\gamma}\left[z^{\beta-\frac{3}{2}}(1-z)^{-\alpha-n}\right] t^{n}=(1-t)^{-\alpha} \mathfrak{D}_{z ; p, v}^{\beta-\gamma}\left[z^{\beta-\frac{3}{2}}\left(1-\frac{z}{1-t}\right)^{-\alpha}\right] .
$$

Thus by applying Theorem 2.3, we obtain the required result.

Theorem 3.2. The following generating relation holds true:

$$
\sum_{n=0}^{\infty} \frac{(\beta)_{n}}{n !}{ }_{2} F_{1 ; p, v}(\delta-n, \beta ; \gamma ; z) t^{n}=(1-t)^{-\beta} F_{1}\left(\alpha, \delta, \beta ; \gamma ;-\frac{z t}{1-t} ; p, v\right),
$$

where $|t|<\frac{1}{1+|t|}, \Re(\delta)>0, \Re(\beta)>0, \Re(\gamma)>\Re(\alpha)>0$.

Proof. Consider the series identity

$$
[1-(1-z) t]^{-\beta}=(1-t)^{-\beta}\left[1+\frac{z t}{1-t}\right]^{-\beta}
$$

Using the power series expansion to the left sides, we have

$$
\sum_{n=0}^{\infty} \frac{(\beta)_{n}}{n !}(1-z)^{n} t^{n}=(1-t)^{-\beta}\left[1-\frac{-z t}{1-t}\right]^{-\beta}
$$

Multiplying both sides of (3.4) by $z^{\alpha-\frac{3}{2}}(1-z)^{-\delta}$ and applying the operator $\mathfrak{D}_{z ; p, v}^{\alpha-\gamma}$ on both sides, we have

$$
\mathfrak{D}_{z ; p, v}^{\alpha-\gamma}\left[\sum_{n=0}^{\infty} \frac{(\beta)_{n}}{n !} z^{\alpha-\frac{3}{2}}(1-z)^{-\delta+n} t^{n}\right]=(1-t)^{-\beta} \mathfrak{D}_{z ; p, v}^{\alpha-\gamma}\left[z^{\alpha-\frac{3}{2}}(1-z)^{-\delta}\left(1-\frac{-z t}{1-t}\right)^{-\beta}\right],
$$

where $\Re(\alpha)>0$ and $|z t|<|1-t|$, thus by Theorem 2.2 , we have

$$
\sum_{n=0}^{\infty} \frac{(\beta)_{n}}{n !} \mathfrak{D}_{z ; p, v}^{\alpha-\gamma}\left[z^{\alpha-\frac{3}{2}}(1-z)^{-\delta+n}\right] t^{n}=(1-t)^{-\beta} \mathfrak{D}_{z ; p, v}^{\alpha-\gamma}\left[z^{\alpha-\frac{3}{2}}(1-z)^{-\delta}\left(1-\frac{-z t}{1-t}\right)^{-\beta}\right] .
$$

Applying Theorem 2.4 on both sides, we get the desired result.

Theorem 3.3. The following result holds true:

$$
\mathfrak{D}_{z ; p, v}^{\eta-\mu}\left[z^{\eta-\frac{3}{2}} E_{\gamma, \delta}^{\mu}(z)\right]=\frac{z^{\mu-2}}{\Gamma(\mu-\eta)} \sum_{n=0}^{\infty} \frac{(\mu)_{n}}{\Gamma(\gamma n+\delta)} \beta_{p, v}(\eta+n, \mu-\eta) \frac{z^{n}}{n !},
$$


FURTHER EXTENSION OF EXTENDED FRACTIONAL DERIVATIVE OPERATOR...

where $\gamma, \delta, \mu \in \mathbb{C}, \Re(p)>0, \Re(q)>0, \Re(\mu)>\Re(\eta)>0, \Re(\lambda)>0, \Re(\rho)>0$ and $E_{\gamma, \delta}^{\mu}(z)$ is Mittag-Leffler function (see [13]) defined as:

$$
E_{\gamma, \delta}^{\mu}(z)=\sum_{n=0}^{\infty} \frac{(\mu)_{n}}{\Gamma(\gamma n+\delta)} \frac{z^{n}}{n !} .
$$

Proof. Using (3.6) in (3.5), we have

$$
\mathfrak{D}_{z ; p, v}^{\eta-\mu}\left[z^{\eta-\frac{3}{2}} E_{\gamma, \delta}^{\mu}(z)\right]=\mathfrak{D}_{z ; p, v}^{\eta-\mu}\left[z^{\eta-\frac{3}{2}}\left\{\sum_{n=0}^{\infty} \frac{(\mu)_{n}}{\Gamma(\gamma n+\delta)} \frac{z^{n}}{n !}\right\}\right] .
$$

By Theorem 2.2, we have

$$
\mathfrak{D}_{z ; p, v}^{\eta-\mu}\left[z^{\eta-\frac{3}{2}} E_{\gamma, \delta}^{\mu}(z)\right]=\sum_{n=0}^{\infty} \frac{(\mu)_{n}}{\Gamma(\gamma n+\delta)}\left\{\mathfrak{D}_{z ; p, v}^{\eta-\mu}\left[z^{\eta+n-\frac{3}{2}}\right]\right\}
$$

Applying Theorem 2.1, we get the required proof.

Theorem 3.4. The following result holds true:

$$
\begin{aligned}
\mathfrak{D}_{z ; p, v}^{\eta-\mu}\left\{z^{\eta-\frac{3}{2}}{ }_{m} \Psi_{n}\left[\begin{array}{c}
\left(\alpha_{i}, A_{i}\right)_{1, m} ; \\
\left(\beta_{j}, B_{j}\right)_{1, n} ;
\end{array}\right]\right\} & =\frac{z^{\mu-2}}{\Gamma(\mu-\eta)} \\
& \times \sum_{k=0}^{\infty} \frac{\prod_{i=1}^{m} \Gamma\left(\alpha_{i}+A_{i} k\right)}{\prod_{j=1}^{n} \Gamma\left(\beta_{j}+B_{j} k\right.} \beta_{p, v}^{\lambda, \rho}(\eta+k, \mu-\eta) \frac{z^{k}}{k !},
\end{aligned}
$$

where $\Re(p)>0, \Re(q)>0, \Re(\mu)>\Re(\eta)>0, \Re(\lambda)>0, \Re(\rho)>0$ and ${ }_{m} \Psi_{n}(z)$ denotes Fox-Wright function defined by (see [7], pp. 56-58)

$$
{ }_{m} \Psi_{n}(z)={ }_{m} \Psi_{n}\left[\begin{array}{c}
\left(\alpha_{i}, A_{i}\right)_{1, m} ; \\
\left(\beta_{j}, B_{j}\right)_{1, n} ;
\end{array}\right]=\sum_{k=0}^{\infty} \frac{\prod_{i=1}^{m} \Gamma\left(\alpha_{i}+A_{i} k\right)}{\prod_{j=1}^{n} \Gamma\left(\beta_{j}+B_{j} k\right.} \frac{z^{k}}{k !} .
$$

Proof. Applying Theorem 2.1 and followed the same procedure used in Theorem 3.3, we get the desired result.

\section{Concluding REMARKS}

In this paper, we established the extension of extended fractional derivative operator. We conclude that when $v=0$ and using the fact that $K_{\frac{1}{2}}(z)=\sqrt{\frac{\pi}{2 z}} e^{-z}$ then all the results established in this paper will reduce to the results obtained by Kiymaz et al. see [6].Also, when $p=v=0$ then we get the results related to the classical Reimann-Liouville fractional derivative operator. 


\section{REFERENCES}

[1] D. Baleanu, P. Agarwal, R. K. Parmar, M. M. Alquarashi, S. Salahshour, Extension of the fractional derivative operator of the Riemann-Liouville, J. Nonlinear Sci. Appl., 10(2017), 2914-2924.

[2] M. A. Chaudhry, A. Qadir, M. Rafique, S. M. Zubair, Extension of Eulers beta function, J. Comput. Appl. Math. 78 (1997) 1932.

[3] M. A. Chaudhry, A. Qadir, H. M. Srivastava and R. B. Paris, Extended Hypergeometric and Confluent Hypergeometric functions, Appl. Math. Comput., 159 (2004) 589-602

[4] J. Choi, A. K. Rathie, R. K. Parmar, Extension of extended beta, hypergeometric and confluent hypergeometric functions, Honam Mathematical J. 36 (2014), No. 2, pp. 357-385.

[5] S. A. Dar, R. B. Paris, A ( $p$,$) -extension of the Appell function F_{1}($.$) and its properties,$ arXiv:1711.07780 [math.CA], 2017.

[6] I. O. Kiymaz, A. Cetinkaya, P. Agarwal, An extension of Caputo fractional derivative operator and iyts application, J. Nonlinear Sci. Appl., 9 (2016), 3611-3621.

[7] A. A. Kilbas, H. M. Sarivastava, J. J. Trujillo, Theory and application of fractional differential equation, North-Holland Mathematics Studies, Elsevier Sciences B.V., Amsterdam, (2006).

[8] M. J. Luo, G. V. Milovanovic, P. Agarwal, Some results on the extended beta and extended hypergeometric functions, Appl. Math. Comput., 248 (2014), 631-651.

[9] S. Mubeen, G. Rahman, K. S. Nisar, J. Choi. M. Arshad, An extended beta function and its properties, Far East Journal of Mathematical Sciences, 102(2017), 1545-1557.

[10] M. A. Özerslan, E. Özergin, Some generating relations for extended hypergeometric functions via generalized fractional derivative operator, Mathematical and Computer Modelling, 52 (2010), 1825-1833.

[11] M. A. Özerslan, E. Özergin, Extension of gamma, beta and hypergeometric functions, J. Comput. Appl. Math. 235 (2011), 4601-4610.

[12] F. W. J. Olver, D. W. Lozier, R. F. Boisvert, C. W. Clark, (eds.), NIST Handbook of Mathematical Functions, Cambridge University Press, Cambridge, 2010.

[13] T. R. Prabhakar, A singular integral equation with a generalized Mittag-Leffler function in the kernel, Yokohama Math. J., 19(1971), 7-15.

[14] R. K. Parmar, P. Chopra, R. B. Paris, On an extension of extended beta and hypergeometric functions, arXiv:1502.06200 [math.CA], 22, 2015. [to appear in J. Classical Anal.]

[15] E. D. Rainville, Special functions, The Macmillan Company, New York, 1960.

[16] G. Rahman, S. Mubeen, K. S. Nisar, J. Choi, Extended special functions and fractional integral operator via an extended Beta function, Submitted.

[17] H. M. Srivastava, R. K. Parmar, P. Chopra, A class of extended fractional derivative operators and associated generating relations involving hypergeometric functions, Axioms, 1 (2012), 238-258. 
FURTHER EXTENSION OF EXTENDED FRACTIONAL DERIVATIVE OPERATOR...

Gauhar Rahman: Department of Mathematics, International Islamic University, IsLAMABAD, PAKISTAN

E-mail address: gauhar55uom@gmail.com

Shahid Mubeen: Department of Mathematics, University of Sargodha, Sargodha, PakISTAN

E-mail address: smjhanda@gmail.com

Kottakkaran Sooppy Nisar: Department of Mathematics, College of Arts and ScienceWadi Aldawaser, 11991, Prince Sattam bin Abdulaziz University, Alkharj, Kingdom of SAUDi ARABIA

E-mail address: n.sooppy@psau.edu.sa; ksnisar1@gmail.com 